\title{
Self-Compensating Displacement Sensor Based on Hydramatic Structured Transducer and Fiber Bragg Grating
}

\author{
Shimeng CHEN, Yun LIU, Xiuxin LIU, Yang ZHANG, and Wei PENG* \\ College of Physics and Optoelectronics Engineering, Dalian University of Technology, Dalian, 116024, China \\ "Corresponding author: Wei PENGＥ-mail: wpeng@dlut.edu.cn
}

\begin{abstract}
An optical fiber displacement sensor with a large measuring range for simultaneous displacement and temperature measurement is presented in this paper. We developed a specific transducer based on the piston and hydraumatic structure to realize a large displacement measurement, which combined the large measuring range and high precision into a single sensor system. The spectrum showed two reflection peaks used to compensate for cross-sensitivity in the displacement detection. This displacement sensor can linearly work in a large measuring displacement range greater than $45 \mathrm{~mm}$ with a high sensitivity of $0.036 \mathrm{~nm} / \mathrm{mm}$. The sensor we reported can be developed for real-time displacement monitoring in many industrial environments such as the mechanical shape or liquid level monitoring.
\end{abstract}

Keywords: Hydraumatic structured transducer, large measuring range, temperature compensating, optical fiber sensor, displacement

Citation: Shimeng CHEN, Yun LIU, Xiuxin LIU, Yang ZHANG, and Wei PENG, "Self-Compensating Displacement Sensor Based on Hydramatic Structured Transducer and Fiber Bragg Grating," Photonic Sensors, 2015, 5(4): 351-356.

\section{Introduction}

Optical fiber sensors have emerged as common sensing elements for temperature [1], strain [2], refractive index (RI) [3], force [4], and other parameters measurement, because of their features of anti-electromagnetic interference, corrosion resistance, compactness, lightweight, and high sensitivity, etc. $[5,6]$. Being one of the important optical fiber sensors, the fiber displacement sensor has been widely applied. Different types of fiber displacement sensors were demonstrated in the fields of the interferometry [7-9], intensity modulation method [10, 11], and wavelength modulation method based on the fiber Bragg grating (FBG) [12-14]. A fiber displacement sensor based on multimode fiber as a resonant cavity was reported [9]. However, the interferometry has the feature of low wavelength division multiplexing. A mathematical model for the two-fiber intensity modulated displacement sensor was proposed [10], which was used to simulate the response of the sensor with different inclined fiber angles. Nevertheless, the strain and temperature cross-sensitivity problem could influence the experimental result. To solve this problem, some technologies were proposed. A type of improved FBG sensors was reported [13]. It could simultaneously measure displacement and temperature. And the displacement sensing process can be tuned by applying the bilateral cantilever beam. Although the problems of low sensitivity and cross-sensitivity have been solved, most of those displacement sensors are limited by small measuring

Received: 31 August 2015 / Revised: 5 September 2015

C The Author(s) 2015. This article is published with open access at Springerlink.com

DOI: $10.1007 / \mathrm{s} 13320-015-0278-4$

Article type: Regular 
ranges and poor durability. In this paper, we develop an optical fiber displacement sensor. The transducer we designed was constructed by a hydraulic transmission system, which could provide a large displacement measuring range. The dual-wavelength FBG written on a joint point of a single mode fiber (SMF) and a photosensitive fiber (PSF) provides two separate FBG wavelengths for temperature compensation. The sensor we designed featured a large measuring range, high sensitivity, favorable durability and could avoid the cross-sensitivity problem.

\section{Principle and system construction}

The structure schematic of the displacement sensor based on the hydramatic structured transducer is shown in Fig. 1. The proposed transducer is composed by two oil columns and two pistons with different cross sectional areas. The transducer can transform the large-displacement into the micro-displacement applied on the cantilever beam. The transducer is a hydraulic transmission system based on the Pascal's principle and can transmit displacement, speed, and power.

Figure 2 illustrates schematics of the optical fiber displacement sensing system. Figure 2(a) shows a sensing system for displacement measurement. The light from a broadband light source (BBS) is launched into the FBG through the coupler, the backward light enters into an optical spectrum analyzer (OSA, AQ6370, YOKOGAWA) through a $3-\mathrm{dB} 2 \times 2$ coupler, and the sensor is attached on a cantilever beam. The reflection spectrum of the dual-wavelength FBG is shown in Fig. 2(b). Because the FBG is written by using $193 \mathrm{~nm}$ excimer laser through a $1065 \mathrm{~nm}$ mask on a joint point of the SMF and PSF, two reflection peaks with different central wavelengths can be observed. Both of the two FBG wavelengths can be tuned by physical parameters, thus we use it for simultaneous displacement and temperature measurement.

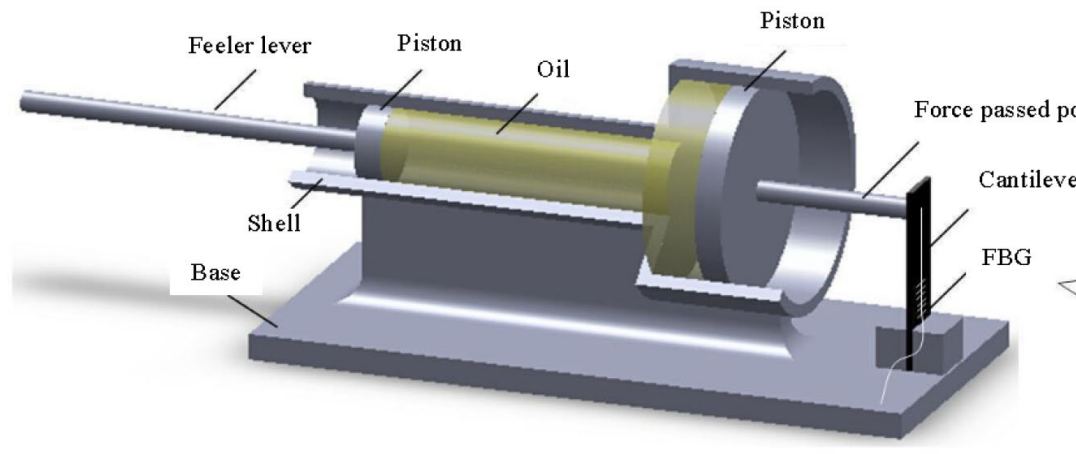

(a)

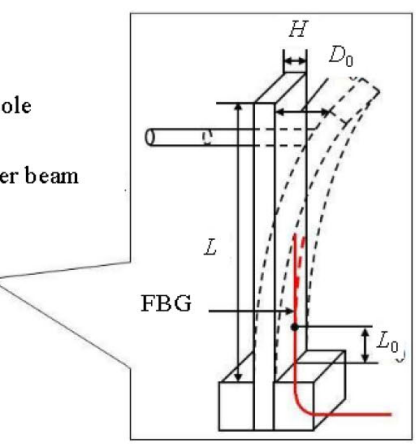

(b) FBG.

Fig. 1 Experimental setup of the measuring system: (a) transducer based on hydramatic structure and (b) cantilever beam attached

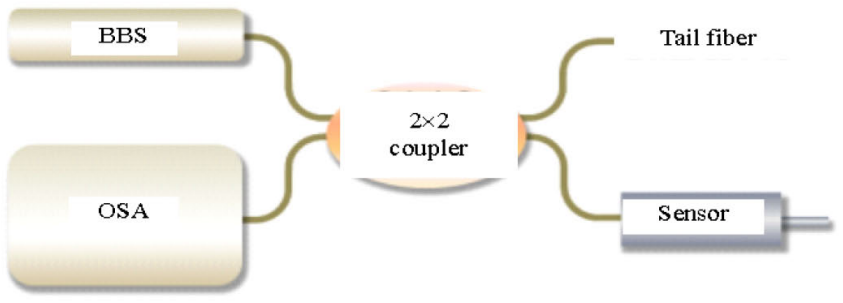

(a)

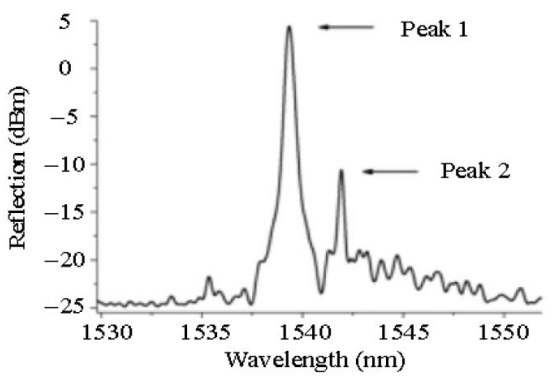

(b)

Fig. 2 Schematics of the optical fiber displacement sensing system: (a) sensing system for displacement measurement and (b) dual-wavelength FBG reflection spectrum. 
According to the law of volume constancy, the hydramatic transducer can transfer largedisplacement $D$ into micro-displacement $D_{0}$ by the area difference between two pistons in their respective working chambers, and the relationship can be expressed as

$$
S \cdot D=S_{0} \cdot D_{0}
$$

where $S$ and $S_{0}$ are the cross section areas of two oil columns. Because the pressures of liquid are equal everywhere, the pressure ratio of two pistons is the same as the area ratio of two pistons, namely the expression is

$$
P=P_{0}=F / S=F_{0} / S_{0}
$$

where $P, P_{0}, F$, and $F_{0}$ are pressures and forces of two pistons, respectively. In our experimental setup, the pressure in the feeler lever is $F=F_{0} / 10$ (the range of $F$ is from $7 \mathrm{~N}$ to $8 \mathrm{~N}$ ) because $S: S_{0}=1: 10$. In addition, we can get less $F$ by increasing the cross section area ratio.

The cantilever beam is shown in Fig. 1(b). When the free end of the transducer produces a displacement of $D_{0}$, it will lead to the cantilever beam to be bent. Then, one side of the beam produces tensile strain while the other side of the beam produces compressing strain. According to the previous work [15], assuming $\lambda_{1}$ and $\lambda_{2}$ are the Bragg wavelengths of the FBG, we find the relationship between the wavelength-shift and displacement of the free end is

$$
\left(\begin{array}{c}
\Delta \lambda_{1} \\
\Delta \lambda_{2}
\end{array}\right)=\left(\begin{array}{ll}
\Omega_{D_{1}} & \Omega_{T_{1}} \\
\Omega_{D_{2}} & \Omega_{T_{2}}
\end{array}\right)\left(\begin{array}{c}
\Delta D \\
\Delta T
\end{array}\right)
$$

where $\Omega D_{i}=\partial \lambda_{i} / \partial D_{i}, \Omega T_{i}=\partial \lambda_{i} / \partial T_{i}(i=1,2)$. As the sensing element (FBG) is composed by different fibers, the deformation responses caused by the displacement are different $\left(\Omega D_{1} \neq \Omega D_{2}\right)$ while the relationship of temperature response is $\Omega T_{1} \approx \Omega T_{2}$. Thus the difference of two peaks' wavelength shifts $d \Delta \lambda=\Delta \lambda_{1}-\Delta \lambda_{2}$ is independent of temperature. Assuming $d \Delta \lambda_{0}=\Delta \lambda_{10}-\Delta \lambda_{20}$, the relationship between the displacement and wavelength is

$$
d \Delta \lambda-d \Delta \lambda_{0}=\left|\Omega D_{1}-\Omega D_{2}\right| \Delta D .
$$

Then, the displacement can be expressed as

$$
\Delta D=\left(d \Delta \lambda-d \Delta \lambda_{0}\right) /\left|\Omega D_{1}-\Omega D_{2}\right| .
$$

As a result, through monitoring the difference between two FBG wavelength shifts $d \Delta \lambda$, the displacement of the free end (the measured displacement value $\Delta D$ ) can be measured.

\section{Experiments and discussion}

In Fig. 1(b), the feeler lever transmits the displacement from the measured object to the free end of the cantilever beam. The length of the beam $L$ was $70 \mathrm{~mm}$, the thickness $H$ was $1 \mathrm{~mm}$, and the FBG was pasted at the place $L_{0}=5 \mathrm{~mm}$. The lengths of FBGs written in the SMF and PSF were $1 \mathrm{~cm}$ and $3 \mathrm{~mm}$, respectively. The reflective peaks of FBGs written in the SMF and PSF were $1539.188 \mathrm{~nm}$ and $1541.624 \mathrm{~nm}$ (Peak 1 and Peak 2 as follows). Using the above structure, we made the following displacement measurement at room temperature $\left(20{ }^{\circ} \mathrm{C}\right)$. The displacements of the free end were from $0 \mathrm{~mm}$ to $45 \mathrm{~mm}$ with a step of $5 \mathrm{~mm}$, and the measured results are plotted in Fig. 3. As the responses of the two Bragg wavelengths decrease linearly, respectively, Peak 1 shifts to the short wavelength at a faster rate than Peak 2 , and the experimental values of $\Omega D_{1}$ and $\Omega D_{2}$ were $-0.049 \mathrm{~nm} / \mathrm{mm} \quad\left(R^{2}=0.991\right)$ and $0.013 \quad \mathrm{~nm} / \mathrm{mm}$ $\left(R^{2}=0.979\right)$ over the range from $0 \mathrm{~mm}$ to $45 \mathrm{~mm}$.

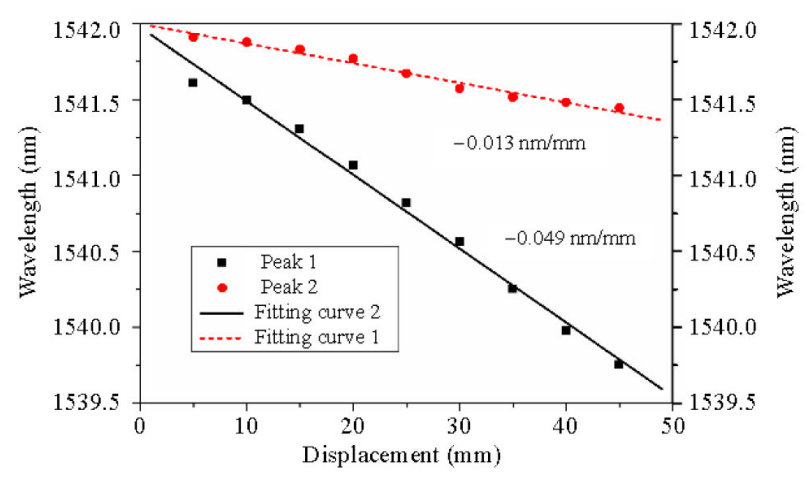

Fig. 3 Responses of dual-wavelength FBG to displacement.

To make sure the measurement result is reliable, the responses of the two Bragg wavelengths of three measurements are shown in Fig. 4, from which we can find the measurement results of wavelength responses to displacement possess good repeatability. 
The standard deviations of Peak 1 and Peak 2 were less than 0.084 and 0.064 , which indicated the experiment results measured in three rounds had little difference, respectively.

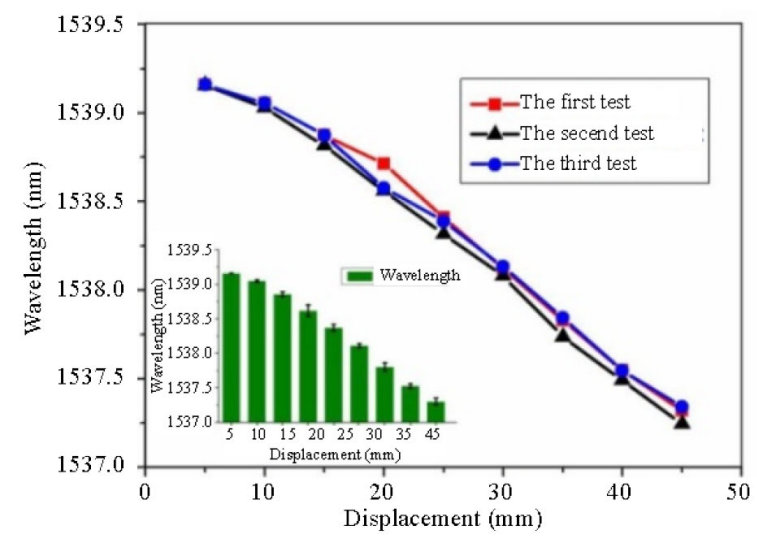

(a)

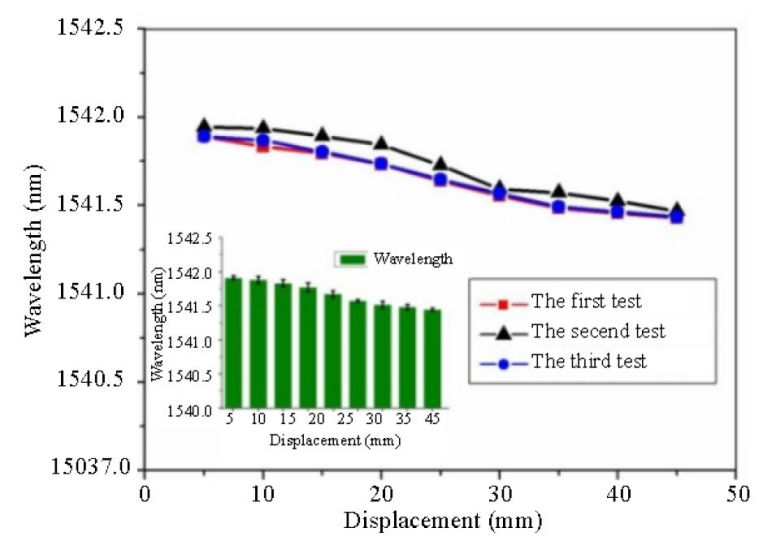

(b)

Fig. 4 Repeatability testing of two FBG wavelengths response to different displacements: (a) Peak 1 and (b) Peak 2.

Then, we tested the temperature characteristic of the sensing element in the water bath over a range from $25{ }^{\circ} \mathrm{C}$ to $65{ }^{\circ} \mathrm{C}$. As shown in Fig. 5 , the two Bragg wavelengths increase with temperature linearly. The experimental values of $\Omega T_{1}$ and $\Omega T_{2}$ were $0.036 \mathrm{~nm} /{ }^{\circ} \mathrm{C} \quad\left(R^{2}=0.995\right)$ and $0.034 \mathrm{~nm} /{ }^{\circ} \mathrm{C}$ $\left(R^{2}=0.991\right)$ during this temperature range.

Thus, as $\Omega D_{1}=-0.049 \mathrm{~nm} / \mathrm{mm}, \Omega D_{2}=$ $-0.013 \mathrm{~nm} / \mathrm{mm}$, and $d \Delta \lambda_{0}=2.436 \mathrm{~nm}$, we obtain

$$
\begin{gathered}
|d \Delta \lambda-2.436|=0.036 \Delta D \times 10^{-6} \\
\Delta D=27.778|d \Delta \lambda-2.436| \times 10^{6} .
\end{gathered}
$$

Therefore, we can get the displacement from the difference of two FBG wavelength shifts. According to $\left(4^{\prime}\right)$ and $\left(5^{\prime}\right)$, we can get the sensor sensitivity of
$0.036 \mathrm{~nm} / \mathrm{mm}$ and the displacement constant of $27.778 \mathrm{~mm} / \mathrm{nm}$.

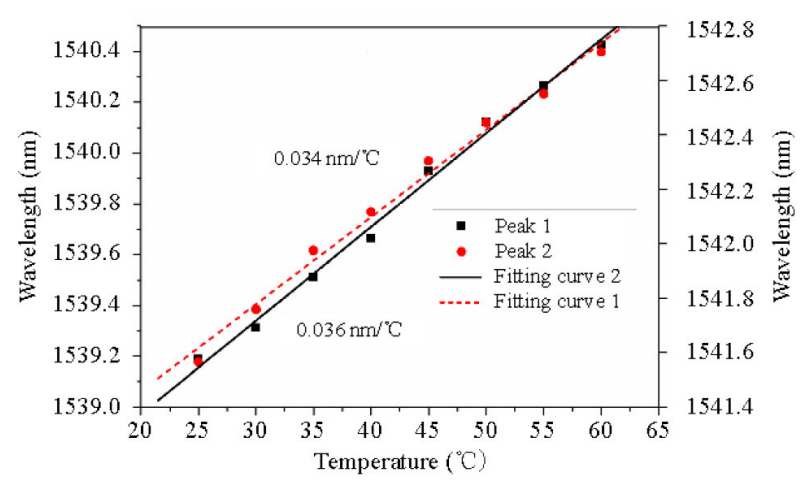

Fig. 5 Responses of dual-wavelength FBG to temperature.

Shen et al. [16] proposed fikr -optic displacement sensor based on the reflective intensity modulated technology for the wide range measurement. The sensing range was over $30 \mathrm{~cm}$, which was over 100 times that of the conventional fiber-optic displacement sensor based on the normal single-mode fiber. However, the reliability and stability of the sensor were limited by the fluctuation of the light source and ifluences of environment. Chen et al. [17] proposed a high-sensitivity fiber-optic displacement sensor fabricated by concatenating two core-offset joints with a separation length of $13 \mathrm{~mm}$ using a commercial fusion splicer. The displacement sensitivities obtained were up to $0.835 \mathrm{~nm} / \mu \mathrm{m}$ and $0.227 \mathrm{~nm} / \mu \mathrm{m}$ in the ranges of $350 \mu \mathrm{m}$ to $1000 \mu \mathrm{m}$, respectively. However, it is hard to be used for the large range displacement measurement. As compared, we proposed an FBG displacement sensor with a good tradeoff between the sensitivity and measurement range, which could linearly work in a large measuring displacement range greater than $45 \mathrm{~mm}$ with a high sensitivity of $0.036 \mathrm{~nm} / \mathrm{mm}$.

To verify the validity of (5'), we chose randomly five testing points, which were $\left(8 \mathrm{~mm}, 27^{\circ} \mathrm{C}\right)$, $\left(16 \mathrm{~mm}, 23{ }^{\circ} \mathrm{C}\right),\left(24 \mathrm{~mm}, 35^{\circ} \mathrm{C}\right),\left(32 \mathrm{~mm}, 51{ }^{\circ} \mathrm{C}\right)$, and $\left(40 \mathrm{~mm}, 31{ }^{\circ} \mathrm{C}\right.$ ). The theoretical curve of the relationship between $\Delta D$ and $d \Delta \lambda$ is shown in Fig.6. Among them, $d \Delta \lambda$ was measured in the experiment. 
We then calculated the displacement constant $(30.591 \mathrm{~mm} / \mathrm{nm})$ which corresponded to the deviation from the theoretical values $(27.778 \mathrm{~mm} / \mathrm{nm})$ of $\pm 5.063 \%$.

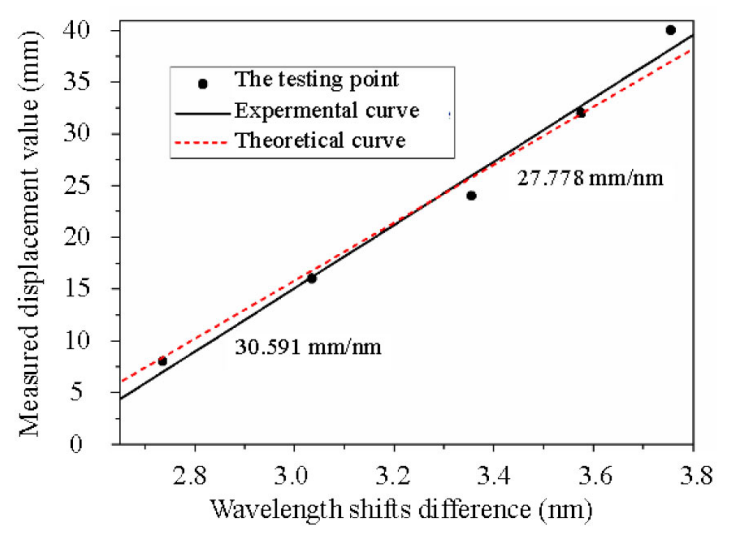

Fig. 6 Relationship between the measured displacement value $(\Delta D)$ and wavelength shift difference $(d \Delta \lambda)$.

\section{Conclusions}

In summary, we experimentally demonstrated a self-compensating displacement sensor with the large measuring range. Because it combined the hydramatic structured transducer and FBG into a single sensor system, it possessed the advantages of both types. We demonstrated that it could make linear displacement measurement up to $45 \mathrm{~mm}$ with a sensitivity of $0.036 \mathrm{~nm} / \mathrm{mm}$. This novel optical fiber displacement sensor has the advantages of the large measuring range, high sensitivity, good durability, and ease to manufacture.

\section{Acknowledgment}

The authors would like to thank financial supports from the National Natural Science Foundation of China (Grant Nos. 61137005 and 11474043) and the Ministry of Education of China (Grant No. DUT14ZD211 and SRFDP 20120041110040).

Open Access This article is distributed under the terms of the Creative Commons Attribution License which permits any use, distribution, and reproduction in any medium, provided the original author(s) and source are credited.

\section{References}

[1] C. E. Lee and H. F. Taylor, "Fiber-optic Fabry-Perot temperature sensor using a low-coherence light source," Journal of Lightwave Technology, 1991, 9(1): 129-134.

[2] B. O. Guan, H. Y. Tam, S. L. Ho, W. H. Chung, and X. Y. Dong, "Simultaneous strain and temperature measurement using a single fibre Bragg grating," Electronics Letters, 2000, 36(12): 1018-1019.

[3] A. Singh, "Long period fiber grating based refractive index sensor with enhanced sensitivity using michelson interferometric arrangement," Photonic Sensors, 2015, 5(2): 172- 179

[4] T. Guo, H. Zhang, B. Liu, G. Li, Q. Zhao, and $\mathrm{X}$. Dong, "Gaussian-strain-chirped fiber Bragg grating couple for temperature-insensitive and intensity-referenced force measurement," IEEE Sensors Journal, 2007, 7(10): 1390- 1394.

[5] H. Li, D. Sheng, and S. Bing, "Recent applications of fiber optic sensors to health monitoring in civil engineering," Engineering Structures, 2004, 26(11): $1647-1657$.

[6] W. R. Habel and K. Krebber, "Fiber-optic sensor applications in civil and geotechnical engineering," Photonic Sensors, 2011, 1(3): 268-280.

[7] T. Y. Wang, S. X. Zheng, and Z. G. Yang, "A high precision displacement sensor using a low-finesse fiber-optic Fabry-Pérot interferometer," Sensors and Actuators A: Physical, 1998, 69(2): 134-138.

[8] F. Ruan, Y. Zhou, Y. L. Lam, S. H. Mei, C. Y. Liaw, and J. Liu, "A precision fiber optic displacement sensor based on reciprocal interferometry," Optics Communications, 2000, 176(1): 105-112.

[9] A. Mehta, W. Mohammed, and E. G. Johnson, "Multimode interference-based fiber-optic displacement sensor," IEEE Photonics Technology Letters, 2003, 15(8): 1129-1131.

[10] P. B. Buchade and A. D. Shaligram, "Simulation and experimental studies of inclined two fiber displacement sensor," Sensors and Actuators A: Physical, 2006, 128(2): 312-316.

[11] H. M. Cao, Y. P. Chen, Z. D. Zhou, and G. Zhang, "Theoretical and experimental study on the optical fiber bundle displacement sensors," Sensors and Actuators A: Physical, 2007, 136(2): 580-587.

[12] X. Dong, X. Yang, C. Zhao, L. Ding, P. Shum, and N. Q. Ngo, "A novel temperature-insensitive fiber Bragg grating sensor for displacement measurement," Smart Materials and Structures, 2005, 14(2): 7-10.

[13] W. G. Zhang, X. Y. Dong, Q. D. Zhao, G. Y. Kai, 
and S. Z. Yuan, "FBG-type sensor for simultaneous measurement of force (or displacement) and temperature based on bilateral cantilever beam," IEEE Photonics Technology Letters, 2001, 13(12): $1340-1342$.

[14] Y. Zhao, H. Zhao, X. Zhang, Q. Meng, and B. Yuan, "A novel double-arched-beam-based fiber Bragg grating sensor for displacement measurement," IEEE Photonics Technology Letters, 2008, 20(15): 1296-1298.

[15] B. O. Guan, H. Y. Tam, S. L. Ho, W. H. Chung, and $\mathrm{X}$. Y. Dong, "Simultaneous strain and temperature measurement using a single fibre Bragg grating," Electronics Letters, 2000, 36(12): 1018-1019.

[16] W. Shen, X. Wu, H. Meng, G. Zhang, and X. Huang, "Long distance fiber-optic displacement sensor based on fiber collimator," Review of Scientific Instruments, 2010, 81(12): 123104.

[17] J. Chen, J. Zhou, and Z. Jia, "High-sensitivity displacement sensor based on a bent fiber Mach-Zehnder interferometer," IEEE Photonics Technology Letters, 2013, 25(23): 2354-2357. 